International Journal of Pure and Applied Mathematics

Volume $97 \quad$ No. 2 2014, 201-210

ISSN: 1311-8080 (printed version); ISSN: 1314-3395 (on-line version)

url: http://www.ijpam.eu

doi: http://dx.doi.org/10.12732/ijpam.v97i2.8

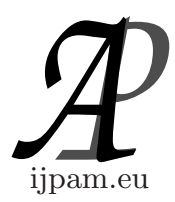

\title{
QUASI-INJECTIVE NEAR-RING GROUPS
}

\author{
Navalakhi Hazarika ${ }^{1}$, Helen K. Saikia ${ }^{2}$ \\ ${ }^{1}$ Department of Mathematics \\ Royal School of Engineering and Technology \\ Guwahati, 781035, INDIA \\ ${ }^{2}$ Department of Mathematics \\ Gauhati University \\ Guwahati, 781014, INDIA
}

\begin{abstract}
We extend the concepts of quasi-injective modules and their endomorphism rings to near-ring groups. We attempt to derive the near-ring character of the set of endomorphism of quasi-injective $N$-groups under certain conditions and this leads us to a near-ring group structure which motivates us to study various characteristics of the structure. If $E$ is a quasi-injective N-group and $S=E n d($ injective hull of $E$ ) then we study the structure $E S$ and various properties of $E S$. It is proved that $E S$ is a minimal quasi-injective extension of $E$ and any two minimal quasi-injective extensions are equivalent. This structure motivates to study the Jacobson radical of endomorphism near-ring of quasi-injective $N$-group $E$. It is established that the near-ring modulo the Jacobson radical is a regular near-ring. Some properties of quasi-injective $N$ groups relating essentially closed $N$-subgroups and complement $N$-subgroups are established.
\end{abstract}

AMS Subject Classification: $16 \mathrm{Y} 30$

Key Words: near-ring groups, quasi-injective $N$-subgroups, essentially closed $N$-subgroups

Received: June 19, 2014

(C) 2014 Academic Publications, Ltd. url: www.acadpubl.eu

${ }^{\S}$ Correspondence author 


\section{Prerequisites}

All basic concepts used in this paper are available in Pilz [4].In this section we define the basic terms and results that are needed for the sequel.

Definition 1.1. For a right near-ring $(N,+,$.$) and a corresponding N$ group $E$, suppose there is an $x \in E$ such that $\{n x \mid n \in N\}=E$. Then $E$ is a monogenic $N$-group and $x$ is a generator.

Definition 1.2. An $N$-subgroup $B$ of $E$ is called fully invariant if for each $N$-homomorphism $f: E \rightarrow E, f(B) \subset B$.

Definition 1.3. A left ideal $A$ of $N$ is called small (strictly small) if $N=B$ for each left ideal ( $N$-subgroup) B such that $N=A+B$.

Since every left ideal is a left $N$-subgroup, a strictly small left ideal of $N$ is also a small left ideal of $N$.

Definition 1.4. The intersection of all maximal ideals maximal as $N$ subgroups of $N$-group $E$ is called radical of $E$ and is denoted by $\mathrm{J}(\mathrm{E})$.

Lemma 1.1. [3]: If the radical ideal $J(N)$ is strictly small in $N$ then the following conditions are equivalent- (i) $Y \in J(N)$ (ii) 1-xy is left invertble for all $x \in N$ (iii) yM=0 for any irreducible left $N$-group $M$.

Definition 1.5. An $N$-subgroup (ideal) I of $E$ is said to be a essentially closed N-subgroup (ideal) of $E$ if I has no proper essential extension in $E$.

Definition 1.6. An $N$-subgroup (ideal) I of $E$ is said to be a essentially closed $N$-subgroup (ideal) of $E$ if I has no proper essential extension in E.

Theorem 1.1. An $N$-group $E$ is quasi-injective if and only if $E$ is fully invariant $N$-subgroup of its injective hull.

Theorem 1.2. If $E$ is quasi-injective then its direct summands are also quasi-injective.

Proof of Theorem 1.1, Theorem 1.2 are given in K. R. Goodearl [6].

Theorem 1.3. [Clay]: For a near-ring $(N,+,$.$) with identity 1$, suppose $E$ is a monogenic unitary $N$-group with generator $x$ and suppose that $T=\{m \in$ $N / \operatorname{Ann}(x) m \in A n n(x)\}$ is a subgroup of $(N,+,$.$) . Then the N$-endomorphisms $E$ of $N$-group $E$ forms a right near-ring where $(f \oplus g)(x)=f(x)+g(x)$ and $(f . g)(x)=f(g(x))$. Also $E$ is an $E_{n d}$ E-group defined by $\phi: E \times E_{n d} E \rightarrow E$ by $\phi(m . f)=m . f=f(m)$. 


\section{Endomorphism Near Ring of Quasi-Injective N-Groups}

In this section we investigate various characteristics of endomorphism near-ring of quasi-injective $N$-groups. We also study Jacobson radical of endomorphism near-ring of quasi-injective $N$-groups. Throughout this section unless and otherwise mention we assume $E$ satisfies the condition of theorem 1.3 and $N$ is a dgnr.

If $\hat{E}$ is injective hull of $E$ and $S=E n d_{N} \hat{E}, \phi: \hat{E} \times S \rightarrow \hat{E}$ by $\phi(m, f)=$ $m . f=f(m), m \in \hat{E}, f \in \mathrm{S}$, then $\hat{E}$ is an $S$-group.

For this $S$-group we get the following:

Proposition 2.1. ES is an $N$-subgroup of $\hat{E}$.

Proof. Let $a, b \in E S$,

$$
a=\sum x_{i} f_{i}, b=\sum y_{j} f_{j}, \quad a-b=\sum x_{i} f_{i}-\sum y_{j} f_{j} \in E S .
$$

Let $n \in N, a \in E S$ to show $n a \in E S$.

$$
\begin{aligned}
n a & =n \sum x_{i} f_{i}=n \sum f_{i}\left(x_{i}\right) \\
& =\left(s_{1}+s_{2}+s_{3}+\cdots+s_{n}\right) \sum f_{i}\left(x_{i}\right) \\
& =s_{1} \sum f_{i}\left(x_{i}\right)+s_{2} \sum f_{i}\left(x_{i}\right)+s_{3} \sum f_{i}\left(x_{i}\right)+\cdots+s_{n} \sum f_{i}\left(x_{i}\right) \\
& =\sum s_{1} f_{i}\left(x_{i}\right)+\sum s_{2} f_{i}\left(x_{i}\right)+\sum s_{3} f_{i}\left(x_{i}\right)+\cdots+\sum s_{n} f_{i}\left(x_{i}\right) \\
& =\sum f_{i}\left(s_{1} x_{i}\right)+\sum f_{i}\left(s_{2} x_{i}\right)+\sum f_{i}\left(s_{3} x_{i}\right)+\cdots+\sum f_{i}\left(s_{n} x_{i}\right) \\
& =\sum\left(s_{1} x_{i}\right) f_{i}+\sum\left(s_{2} x_{i}\right) f_{i}+\sum\left(s_{3} x_{i}\right) f_{i}+\cdots+\sum\left(s_{n} x_{i}\right) f_{i} \in E S,
\end{aligned}
$$

because $\left.\left(s_{j} x_{i}\right) \in E\right)$.

Proposition 2.2. (a) ES is quasi-injective.

(b) $E S$ is the intersection of all quasi-injective $N$-subgroups of $\hat{E}$ containing $E$. So ES is the smallest $N$-subgroup of $\hat{E}$ containing $E$.

(c) $E$ is quasi-injective if and only if $E=E S$.

Proof. (a) Let $M$ be an $N$-subgroup of $E S$ and $f: M \rightarrow E S$. We take the inclusion map $i: E S \rightarrow \hat{E}$. Then the composite map $h=$ if $: M \rightarrow \hat{E}$. Since $\hat{E}$ is injective, so $h$ can be extended by some $\lambda: \hat{E} \rightarrow \hat{E}$ such that for $x \in M, x . \lambda=\lambda(x)=x . h=x$. (if $)=($ if $)(x)=i(f(x))=f(x)=x$.f where 
$x . f=f(x) \in E S$. Thus $f$ is induced by $\lambda \in S$. Now let $g \in S$. Then for $y=\sum x_{i} g_{i} \in E S, \sum\left(x_{i} g_{i}\right) \lambda=\sum x_{i}\left(g_{i} \lambda\right) \in E S$, since $g_{i} \lambda \in S$.

Therefore $(E S) \lambda \subseteq E S$. $\lambda$ induces $\bar{\lambda}: E S \rightarrow E S$. i.e. $\lambda$ can be restricted by some $\bar{\lambda}: E S \rightarrow E S$ such that $x \bar{\lambda}=x . \lambda$ for $x \in E S$. Therefore $x \bar{\lambda}=x . f$ for $x \in M[\because x \lambda=x . f$ for $x \in M$ and $M \subseteq E S]$. i.e. $f$ is induced by $\bar{\lambda}: E S \rightarrow E S \Rightarrow E S$ is quasi-injective.

(b) Let $P$ be any quasi-injective $N$-subgroup of $\hat{E}$ containing $E$. We wish to show $E S=\cap P$. Since by (a) $E S$ is quasi-injective. So $\cap P \subseteq E S$. Now to show $E S \subseteq \cap P$. We will show $E S \subseteq P$, so it is sufficient to show that $P \alpha \subseteq P \quad \forall \alpha \in S$. Since if $\forall \alpha \in S, P \alpha \subseteq P$ then $P S \subseteq P$. But $E \subseteq P \Rightarrow$ $E S \subseteq P S \quad[\because E \subseteq P \Rightarrow E \lambda \subseteq P \lambda] \Rightarrow E S \subseteq P$. To prove this we see that $Q(\alpha)=\{x \in P / x \alpha \in P\}$ is an $N$-subgroup of $P$. Let $x, y \in Q(\alpha) \Rightarrow x \alpha \in$ $P, y \alpha \in P . \quad x \alpha-y \alpha \in P \Rightarrow \alpha(x)-\alpha(y) \in P \Rightarrow \alpha(x-y) \in P \Rightarrow(x-y) \in Q(\alpha)$. Next to show for $n \in N, x \in Q(\alpha), n x \in Q(\alpha) . x \in Q(\alpha) \Rightarrow x \in P$ such that $x . \alpha \in P . \because x \in P, n \in N \Rightarrow n x \in P(\because N P \subseteq P) .(n x) \cdot \alpha=\alpha(n x)=n \alpha(x)=$ $n(x . \alpha) \in P(N P \subseteq P) \Rightarrow n x \in Q(\alpha)$. Therefore $Q(\alpha)$ is an $N$-subgroup of $P$. We have only to show that $Q(\alpha)=P \quad \forall \alpha \in S$, since then $y \in P \Rightarrow y \in Q(\alpha) \Rightarrow$ $y . \alpha \in P \Rightarrow P \alpha \subseteq P$. Since $q \rightarrow q \alpha, q \in Q(\alpha)=Q$ a map of $Q$ into $P$ and since $P$ is quasi-injective, so there exists $\alpha_{1}: P \rightarrow P$ such that $q \alpha_{1}=q \alpha \quad \forall q \in Q$. Since $\hat{E}$ is injective, $\exists \alpha^{\prime} \in S$ such that $x \alpha^{\prime}=x \alpha_{1} \quad \forall x \in P$. Since $P \alpha^{\prime} \subseteq P$. If $P\left(\alpha^{\prime}-\alpha\right)=0$ then $P \alpha^{\prime}=P \alpha$. So $P \alpha \subseteq P$. So if $Q(\alpha) \neq P$ then $P\left(\alpha^{\prime}-\alpha\right) \neq 0$. As we know $E \leq_{e} \hat{E} \Rightarrow P \leq_{e} \hat{E} \quad(\because$ if $A(\neq 0) \leq \hat{E} \& P \cap A=0$ then $E \cap A=0$ contradicts $\left.E \leq_{e} \hat{E}\right)$. Now $P\left(\alpha^{\prime}-\alpha\right)$ is $N$-subgroup of $\hat{E}$. $a, b \in P\left(\alpha^{\prime}-\alpha\right)$. Then let $a=p_{1}\left(\alpha^{\prime}-\alpha\right), b=p_{2}\left(\alpha^{\prime}-\alpha\right) \quad a-b=p_{1}\left(\alpha^{\prime}-\alpha\right)-p_{2}\left(\alpha^{\prime}-\alpha\right)=$ $\left(p_{1}-p_{2}\right)\left(\alpha^{\prime}-\alpha\right) \in P\left(\alpha^{\prime}-\alpha\right) \quad\left(\because\left(\alpha^{\prime}-\alpha\right) \in S\right)$. For $n \in N, x \in P\left(\alpha^{\prime}-\alpha\right)$, let $x=p_{1}\left(\alpha^{\prime}-\alpha\right)$. Now $n p_{1}\left(\alpha^{\prime}-\alpha\right)=n\left(\alpha^{\prime}-\alpha\right) p_{1}=n \alpha^{\prime}\left(p_{1}\right)-n \alpha\left(p_{1}\right)=$ $\alpha^{\prime}\left(n p_{1}\right)-\alpha\left(n p_{1}\right)=\left(\alpha^{\prime}-\alpha\right)\left(n p_{1}\right)=\left(n p_{1}\right)\left(\alpha^{\prime}-\alpha\right) \in P\left(\alpha^{\prime}-\alpha\right)$, Therefore $P\left(\alpha^{\prime}-\alpha\right) N$-subgroup of $\hat{E}$. Consequently we have $P\left(\alpha^{\prime}-\alpha\right) \cap P \neq 0$. But if $x, 0 \neq y \in P$ are such that $y=x\left(\alpha^{\prime}-\alpha\right) \in P\left(\alpha^{\prime}-\alpha\right) \cap P$. Then since $x \alpha^{\prime}=x \alpha_{1}\left[\because x \in P \quad y=x\left(\alpha^{\prime}-\alpha\right)=\left(\alpha^{\prime}-\alpha\right)(x)=\left(\alpha^{\prime} x-\alpha x\right)=x \alpha^{\prime}-x \alpha\right]$ $x \alpha=x \alpha_{1}-y \in P$. Then $x \in Q(\alpha)$ so that $x \alpha=x \alpha^{\prime}$ and so $y=0$, a contradiction. Which establishes (b).

(c) Since $E S$ is the intersection of all quasi-injective $N$-subgroups of $\hat{E}$, containing $E$. $E$ is quasi-injective $\Rightarrow E S \subseteq E$. And $E \subseteq E S$ is obvious by inclusion map: $E S=E$

Definition 2.1. $(P, E, f)$ denotes a $N$-monomorphism $f: E \rightarrow P$ and is called an extension of $E$. An extension $(P, E, f)$ of an $N$-group $E$ is a minimal 
quasi-injective extension in case $P$ is quasi-injective and the following condition is satisfied:

If $(A, E, g)$ is any quasi-injective extension of $E$, then there exists a monomorphism $\phi: P \rightarrow A$ such that:

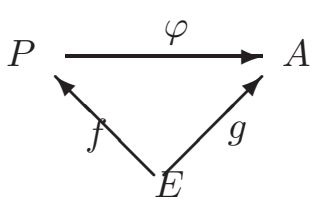

commutes i.e. $g=\phi f$

Proposition 2.3. ES is minimal quasi-injective extension of E. Any Two minimal quasi-injective extensions are equivalent.

Proof. Let $(A, E, g)$ be any quasi-injective extension of $E$. Let $\hat{A}=E(A)$ $\& \Omega=\operatorname{Hom}_{N}(\hat{A}, \hat{A})$. Then by proposition $2.2, A \Omega \subseteq A$. Since $E S$ is an essential extension of $E$, the $N$-monomorphism $g: E \rightarrow \hat{A}$ can be extended to a monomorphism (also denoted by $g$ ) of $E S$ in $\hat{A}$. [Since if $f: A \stackrel{\text { mono }}{\longrightarrow} E, E$ injective, $A \leq_{e} B$, then $f$ extends to $\left.f^{\prime}: B \stackrel{\text { mono }}{\longrightarrow} E\right]$ Since $g(E S)$ is quasiinjective. $[\because g(E S) \cong E S, \because \operatorname{Kerg}=0(f: A \stackrel{\text { mono }}{\longrightarrow} B, A \cong f(A))]$.

Then $(g(E S)) \Omega \subseteq g(E S)$ and we conclude that $(B) \Omega \subseteq B$ where $B=$ $g(E S) \cap A \subseteq g(E S)$, so $g^{-1}(B) \subseteq(E S)[\because A B \subseteq B, A C \subseteq C, A(B \cap C)=$ $A B \cap A C \subseteq B \cap C]$.

Since $B \subseteq(B) \Omega$ is obvious. Therefore by Proposition $2.2 B$ is quasiinjective. It follows that $g^{-1}(B)$ is a quasi-injective extension of $E \subseteq E S$. Since $E S$ is the smallest quasi-injective extension of $E$ contained in $\hat{E}$, we conclude that $g^{-1}(B)=(E S)$. So $B=g(E S) \subseteq A$. This establishes that $E S$ is a minimal quasi-injective extension.

Next if $(A, E, g)$ is also a minimal quasi-injective extension of $E$, then $(A, E, g)$ is also equivalent to $E S$. $E S$ minimal quasi-injective extension of $E$. $(A, E, g)$ is also quasi-injective extension of $E$. By definition for $E \stackrel{\text { mono } \phi}{\longrightarrow}$ $E S, E \stackrel{\text { mono } f}{\longrightarrow} A$, there exists $E S \stackrel{\text { mono } \phi}{\longrightarrow} A$ such that the diagram

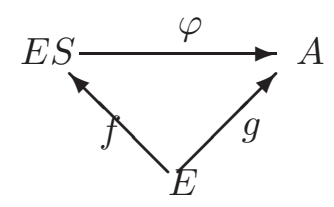


commutes i.e. $g=\phi f$

Again $(A, E, g)$ is minimal quasi-injective extension of $E$. ES is also quasiinjective extension of $E$. By definition for $E \stackrel{\text { monog }}{\longrightarrow} A, E \stackrel{\text { monof }}{\longrightarrow} E S$ there exists $A \stackrel{\text { monow }}{\longrightarrow} E S$ such that the diagram

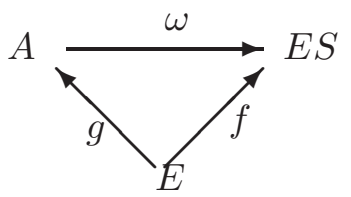

commutes i.e. $f=\omega g$.

Now $f=\omega g \Rightarrow f=\omega \phi f$. So $I=\omega \phi$. Again $g=\phi f \Rightarrow g=\phi \omega g$. So $I=\phi \omega$. Thus $\omega$ and $\phi$ both are invertible which implies both $\omega$ and $\phi$ are isomorphic. Hence $E S \cong A$.

Definition 2.2. A near-ring $N$ is said to be a regular near-ring if for every element $x \in N$, there exists an element $y \in N$ such that $x y x=x$.

Theorem 2.1. Let $E$ be quasi-injective $N$-group let $\Lambda=H o m(E, E)$ and let $J=J(\Lambda)$ denote the Jacobson radical of $\Lambda$ and is strictly small in $\Lambda$. Then $J=\{\lambda \in \Lambda / E$ essential extension of $\operatorname{Ker} \lambda\}$. If for $\gamma \in J, \lambda \in \Lambda, \gamma \lambda \in J$ then $\Lambda / J$ is a regular near-ring. Where addition of two $N$-subgroups is again $N$-subgroup of $E$ and $N$ need not be dgnr.

Proof. Let $I=\{\lambda \in \Lambda / E$ essential extension of $\operatorname{Ker} \lambda\}$.

If $\lambda \in \Lambda, \quad \mu, \gamma \in I$, then $\operatorname{Ker}(\mu+\gamma) \supseteq \operatorname{Ker} \mu \cap \operatorname{Ker} \gamma$. Since $x \in \operatorname{Ker} \mu \cap$ $\operatorname{Ker} \gamma \Rightarrow x \in \operatorname{Ker} \mu \quad \& \quad x \in \operatorname{Ker} \gamma \Rightarrow \mu(x)=0 \& \gamma(x)=0 \Rightarrow(\mu+\gamma)(x)=$ $0 \Rightarrow x \in \operatorname{Ker}(\mu+\gamma)$. Since $\operatorname{Ker} \mu \cap \operatorname{Ker} \gamma$ is an essential $N$-subgroup, therefore $\operatorname{Ker}(\mu+\gamma)$ is an essential $N$-subgroup of $E x \in \operatorname{Ker} \gamma \Rightarrow \gamma(x)=0$. Now for $\mu, \lambda \in \Lambda, \gamma \in I,(\mu(\lambda+\gamma)-\mu \lambda)(x)=(\mu(\lambda+\gamma)(x)-(\mu \lambda)(x)=(\mu \lambda)(x)+$ $0-(\mu \lambda)(x)=0$, since $\gamma(x)=0$. Therefore $x \in \operatorname{Ker}(\mu(\lambda+\gamma)-\mu \lambda)$. And so $\operatorname{Ker} \lambda \subseteq \operatorname{Ker}(\mu(\lambda+\gamma)-\mu \lambda) \Rightarrow(\mu(\lambda+\gamma)-\mu \lambda) \in I$. Therefore $I$ is left ideal of $\Lambda$.

However if $\lambda \in I, \operatorname{Ker}(1+\mu \lambda)=0$ for $\operatorname{Ker} \lambda \cap \operatorname{Ker}(1+\mu \lambda)=0$. For if, $\lambda \in I$ we have $E$ essential extension of $\operatorname{Ker} \lambda . \quad x \in \operatorname{Ker} \lambda \cap \operatorname{Ker}(1+\mu \lambda) \Rightarrow \lambda(x)=0$ and $(1+\mu \lambda)(x)=0 \Rightarrow \mathrm{x}+\mu(\lambda)(x)=0 \Rightarrow x+\mu(0) \Rightarrow x=0$. Again $\lambda \in I \Rightarrow E$ essential extension of $\operatorname{Ker} \lambda \Rightarrow \operatorname{Ker}(1+\mu \lambda)=0$.

$(1+\mu \lambda): E \rightarrow(1+\mu \lambda) E$ is an isomorphism $\Rightarrow \exists g \in \Lambda$ such that $g(1+\mu \lambda)=$ $i$, so $(1+\mu \lambda)$ has a left inverse $\forall \lambda \in I \quad \& \quad \forall \mu \in \Lambda$. So, $\lambda \in J$ [by lemma 1.1]. This establishes that $I \subseteq J$. 
Next let $\lambda$ be arbitrary element of $\Lambda$, let $L$ be a complement N-subgroup of $E$ corresponding to $K=\operatorname{Ker}(\lambda)$ and consider the correspondence $\lambda x \rightarrow x$ $\forall x \in L$. If $\lambda x=\lambda y$ with $x, y \in L$, then $\lambda(x-y)=0$ and then $(x-y) \in K \cap L$ $=0$. Since $E$ is quasi-injective, the map $\lambda x \rightarrow x$ of $\lambda L$ in $L$ is induced by some $\theta \in \Lambda$. If $u=x+y \in L+K, x \in L, y \in K$, then

$$
\begin{aligned}
(\lambda-\lambda \theta \lambda)(u) & =\lambda(x)-\lambda \theta \lambda(x)=\lambda(x)-\lambda(x)=0 \\
& \Rightarrow \lambda-\lambda \theta \lambda=0
\end{aligned}
$$

$[\lambda \theta \lambda(x)=\lambda \theta(x)=\lambda(x)=x$, as for $x \in L \theta(x)=\lambda(x)=x]$.

Since $E \supseteq_{e} L+K$ as $K \subseteq L+K$ and since $\operatorname{Ker}(\lambda-\lambda \theta \lambda) \supseteq L+K$, we conclude that $\lambda-\lambda \theta \lambda \in I$. Now to show $J=I$. If $\lambda \in J$ and $\theta \in \Lambda$ is chosen so that $u=(\lambda-\lambda \theta \lambda) \in I,(1-\theta \lambda)^{-1}$ exists.(Since $J$ is Jacobson Redical. Therefore $(1-\theta \lambda)^{-1}(u)=(1-\theta \lambda)^{-1}(\lambda-\lambda \theta \lambda)=(1-\theta \lambda)^{-1}(1-\theta \lambda) \lambda=\lambda$ and $\lambda \in I[\because I$ is a left ideal]. Thus $J=I$ is asserted. Also $I$ is an ideal by given condition. Thus $\Lambda$ is a regular modulo $I$.

From (1) $\lambda \bar{\theta} \lambda=\bar{\lambda}$ in $\Lambda / I$. Therefore $\Lambda / J$ is regular ring as $J=I$.

\section{Some Properties of Quasi-Injective N-Groups}

This section contains some properties of quasi-injective $N$-groups related to essentially closed $N$-subgroups and complement $N$-subgroups. Let $M$ be an $N$-subgroup of $E$. We consider $F=\{P / P \quad N$-subgroup of $E, P \cap M=0\} . F \neq$ $\Phi,(0) \in F . C=\left\{P_{i} / P_{i} \in F\right\}$ is a chain in $F$. Let $K=\cup P_{i} \cdot\left[x, y \in \cup P_{i} \Rightarrow\right.$ $x \in P_{i}, y \in P_{j}$.Ifi $>J, x, y \in P_{j}$. Therefore $(x-y) \in P_{j} \Rightarrow(x-y) \in \cup P_{i}$. Again $n \in N, x \in \cup P_{i} \Rightarrow x \in P_{j}$ for some $\mathrm{j}$, then $\left.n x \in P_{j} \Rightarrow n x \in \cup P_{i}\right]$ Since $P_{i} \cap M=0 \forall i .\left(\cup_{i} P_{i}\right) \cap M=\cup_{i}\left(P_{i} \cap M\right)=0 \& \cup_{i} P_{i} \leq E$. Therefore $\cup_{i}\left(P_{i} \in C\right.$. So by Zorn's Lemma the $N$-subgroup $K$ is maximal in the set of those $N$-subgroups $P$ satisfying $P \cap N=0$. Then $K$ is said to be complement of $M$ in $E$.

Definition 3.1. The $N$-subgroup $K$ is maximal in the set of those $N$ subgroups $P$ satisfying $P \cap M=0$ is said to be complement of $M$ in $E$. A complement $N$-subgroup(ideal) of $E$ is an $N$-subgroup $A$ which is a complement in $E$ of some $N$-subgroup(ideal) $B$.

If sum of two $N$-subgroups is again an $N$-subgroup of an $N$-group we get the following: 
Lemma 3.1. If $M$ is an $N$-subgroup of $E$ and if $K$ is any complement of $M$ in $E$, then there exists a complement $Q$ of $K$ in $E$ such that $Q \supseteq M$. Furthermore any such $Q$ is a maximal essential extension of $M$ in $E$.

Proof. Let $F=\{I / I \cap K=0, M \subseteq I\}$. Since $M \in F, F \neq \Phi$. Let $C=\left\{C_{i} / i \in \lambda\right.$, גindex, $\left.C_{i} \in F\right\}$ be a chain. $Q=\cup C_{i}$. Now $\left(\cup_{i \in \lambda} C_{i}\right) \cap K=$ $\cup_{i \in \lambda}\left(C_{i} \cap K\right)=0 \quad \forall i . \quad\left[\because C_{i} \cap K=0 \quad \forall i\right] \quad \& \quad M \subset \cup_{i \in \lambda} C_{i} \quad \forall i, M \subseteq C_{i}$. So by Zorn's Lemma $Q \in F$, maximal element exists. Thus $Q$ in the first sentence exists.

Now to prove the second part.

Let $T$ be any non-zero $N$-subgroup of $Q$ and assume that $T \cap M=0$. Since $T \cap K=0 \quad\left[Q \leq_{c} K, T \leq_{s} Q\right]$. Therefore the sum $K_{1}=T+K$ is direct and $K_{1}$ properly contains $K . \because K_{1} \cap M=0 . \quad$ [ If possible let $K_{1} \cap M \neq 0 . \quad K_{1} \cap M=$ $(T+K) \cap M$. Let $t+k=n \in(T+K) \cap M \Rightarrow k \in K \cap(M+T) \subseteq K \cap Q \Rightarrow k=$ $0 \Rightarrow n=t \in M \cap T$ contradiction to $T \cap M=0$. Therefore $K_{1} \cap M=0$.] This contradicts the definition of $K$. This proves that $Q$ is an essential extension of $M$. If $P$ is an $N$-subgroup of $E$ properly containing $Q$, then $P \cap K \neq 0$ and $(P \cap K) \cap M=P \cap(K \cap M)=P \cap 0=0$. Thus $P$ is not essential extension of $M$, completing the proof.

Lemma 3.2. The essentially closed $N$-subgroups of an $N$-group $E$ coincide with the complement $N$-subgroup of $E$. If $M$ and $K$ are complement $N$-subgroups and if $K$ is a complement of $M$ in $E$ then $M$ is a complement of $K$ in $E$.

Proof. Let $M$ be a essentially closed $N$-subgroup and $K$ is any complement of $M$. Then by lemma 3.1 there exists a complement $Q$ of $K$ such that $M \subseteq Q$. This $Q$ is maximal essential extension of $M$ in $E$. But $M$ is essentially closed, so it has no proper essential extension. Therefore $M=Q$ is a complement $N$-subgroup.

Next let $M$ be complement of an $N$-subgroup $P$. Then $\exists$ a complement $K$ of $M$ which contains $P$.

If possible let $M^{\prime} \leq E$ such that $M \subseteq M^{\prime} \quad \& \quad K \cap M^{\prime}=0$. Then $P \cap M^{\prime}=$ 0. $\quad \because P \subset K$, which contradicts (1). Therefore $M$ is also maximal such that $K \cap M=0$. Therefore $M$ is complement of $K$. Then $M$ is essentially closed by lemma3.1. This also proves the last statement.

Theorem 3.1. Let $E$ be quasi-injective and let $M$ be a essentially closed $N$-subgroup, then for each $N$-subgroup $K$ of $E, N$-homomorphism $w: K \Rightarrow M$ can be extended to $N$-homomorphism $u: E \rightarrow M$ 
Proof. Let $F=\{L / w$ is extended to a map of $T$ into $M$ for $N$-subgroup $T$ of $E$ containing $L\}$ By Zorn's lemma we can assume that $K$ is such that $w$ cannot be extended to a map of $T$ into $M$ for any $N$-subgroup $T$ of $E$ which properly contains $K$. Since, $E$ is quasi-injective, $w$ is induced by a map $u: E \rightarrow E \&$ let $L$ complement of $M$ in $E$. Suppose $u(E) \varsubsetneqq M$. Since $M$ is essentially closed. $M$ is a complement of $L$. Therefore, since $u(E)+M \supset M$, we see that $(u(E)+M) \cap L \neq 0$. Let $0 \neq x=a+b \in(u(E)+M) \cap L \Rightarrow a \in$ $u(E), b \in M$. If $a \in M$ then $x \in M \cap L=0$, a contradiction. Therefore $a \notin M$ and $a=x-b \in L+M$. Now $T=y \in E / u(y) \in L+M$ is an $N$-subgroup of $E$ containing $K . \because x \in K \Rightarrow w(k) \in M \Rightarrow u(k) \in M \quad \forall k \in K$. Therefore $T$ contains $K$. If $y \in E$ is such that $u(y)=a$ then $y \in T$, but $y \notin K$ since $a \notin M .[\because y \in T \Rightarrow u(y)=a \in L \& y \in K \Rightarrow w(y) \in M \Rightarrow u(y) \in M \quad \forall y \in K$, contradiction to $a \notin M]$.

Let $\pi$ denote the projection of $L+M$ on $M$. Then $\pi u$ is a map of $T$ in $M$ and $\pi u(y)=u(y)=w(y) \quad \forall y \in K .[\because y \in K \Rightarrow w(y) \in M \Rightarrow u(y) \in M \quad \forall y \in K]$. Thus $\pi u$ is a proper extension of $w$, a contradiction. Therefore $u(E) \subseteq M$, so $u$ is the desired extension.

Corollary 3.1. For quasi-injective $N$-group $E$.

(1) If $M$ is essentially closed $N$-subgroup of $E$, then $M$ is a direct summand of $E$ and $M$ is quasi-injective. Also $M$ has a complement in $E$.

(2) If $P$ is any $N$-subgroup of $E$, then there exists a quasi-injective essential extension of $P$ contained in $E$.

(3) Each minimal quasi-injective extension of an $N$-group $K$ is an essential extension of $K$.

Proof. (1) If $e: E \rightarrow M$ is the extension given by theorem 3.1 of the injection map $M \rightarrow M$ then $E=M \oplus \operatorname{Ker}(e)$ where $e(m)= \begin{cases}m, & m \in M \\ 0, & m \notin M\end{cases}$ So that $M$ is a direct summand of $E$. Therefore $M$ is quasi-injective by theorem 1.2 Moreover $\operatorname{Ker}(e)$ is complement of $M$. Since

$$
M \cap \operatorname{Ker}(e)=(0)
$$

$M$ essentially closed $\Rightarrow M$ complement of some $N$-subgroup $K \Rightarrow K$ is complement of $M$, i.e.

$$
\stackrel{\max }{M} \bigcap^{\max } K=(0)
$$


(2) $\&(3) \Rightarrow \operatorname{Ker}(e) \subset K$. Let $(0 \neq) x \in K \Rightarrow x \notin M \Rightarrow e(x)=0$ [by definition of $e] \Rightarrow x \in \operatorname{Ker}(e)$.

Therefore $K \subset \operatorname{Ker}(e)$. Therefore $K=\operatorname{Ker}(e) \Rightarrow \operatorname{Ker}(e)$ complement of $M$.

(2) Let $F=\left\{I / P \subseteq I, P \leq_{e} I\right\} . P \in F$. Therefore $F \neq \phi$.

Let $\left\{C_{i} / C_{i} \in F\right\}$ be a chain in $F . \quad M=\cup_{i} C_{i}, P \subseteq C_{i} \quad \forall I \Rightarrow P \subseteq$ $\cup_{i} C_{i}, P \leq_{e} C_{i} \quad \forall I \Rightarrow P \leq_{e} \cup_{i} C_{i}\left[P \cap A_{i} \neq 0 \quad \forall i, A_{i} \leq C_{i}\right.$. Since $P \cap\left(\cup_{i} A_{i}\right)=$ $\left.\cup_{i}\left(P \cap A_{i}\right) \neq 0 . \cup A_{i} \leq \cup C_{i}\right]$

If possible $M=\cup_{i} C_{i} \leq_{e} K$. Therefore $P \leq_{e} M \leq_{e} K \Rightarrow P \leq_{e} K$, contradicts maximality of $M$. So by Zorn's Lemma $P$ is contained in essentially closed $N$-subgroup $M$ which is essential extension of $P$ and $M$ is quasi-injective by (1).

(3) Let $A$ be any minimal quasi-injective extension of an $N$-group $K$. Let $K$ is contained in quasi-injective essential extension $B$ by (2). i.e. $B$ essentially closed. So as $B$ is essential extension of $K, A$ is also essential extension of $K$. Thus every minimal quasi-injective extension of an $N$-group $K$ is an essential extension of $K$.

\section{References}

[1] Faith Carl and Utumi Y., Quasi-injective modules and their endomorphism rings, Arch Math., xv(1),(1963), 166-174.

[2] Han Chang, Woo. and Choi, Su-Jeong., Generalizations of the Quasiinjective module, Comm. Korean Math. Soc., 10 (4), (1995), 811-813.

[3] Saikia Helen K. and Misra Kalpana, On p-injective strictly FGD near- ring, Journal of Rajasthan Academy of Physical Sciences, 6(4),(2007), 361-370.

[4] Pilz, G., Near-rings, North Holland publishing Company, Amsterdam (1977).

[5] Goodearl K. R., Ring theory, Nonsingular rings and modules, Maecel Dekker, INC. New York and Basel(1994).

[6] Clay, J.R., Near-rings: Geneses and Applications, Oxford Univ. Press, Oxford(1992). 